\title{
Les collocations et la construction du savoir scientifique
}

\section{Christopher Gledhill}

\section{OpenEdition}

\section{Journals}

Édition électronique

URL : http://journals.openedition.org/asp/2989

DOI : 10.4000/asp.2989

ISSN : 2108-6354

\section{Éditeur}

Groupe d'étude et de recherche en anglais de spécialité

\section{Édition imprimée}

Date de publication : 1 décembre 1997

Pagination : 85-104

ISSN : 1246-8185

\section{Référence électronique}

Christopher Gledhill, «Les collocations et la construction du savoir scientifique », ASp [En ligne], 15-18 | 1997, mis en ligne le 08 mai 2012, consulté le 22 avril 2019. URL : http:// journals.openedition.org/asp/2989; DOI : 10.4000/asp.2989

Ce document a été généré automatiquement le 22 avril 2019

Tous droits réservés 


\title{
Les collocations et la construction du savoir scientifique
}

\author{
Christopher Gledhill
}

\section{Introduction}

1 Pour l'apprenant d'une langue de spécialité et pour le néophyte d'une discipline scientifique quelconque, il est de mise de pouvoir entrer sans difficulté dans le discours de la communauté des spécialistes. Ce problème a été abordé de façon différente selon les traditions. Par exemple, il existe quantité d'études linguistiques sur la rhétorique du discours spécialisé, par exemple sur la structure rhétorique des articles spécialisés (Swales 1990; Nwogu 1989), la reformulation et la vulgarisation (Harvey 1995) ou le discours de l'évaluation (Thomson \& Yiyun 1991). De même, les études terminologiques ont su exploiter l'analyse des corpus informatisés pour quantifier le lexique spécialisé (Knowles 1997) et pour suivre la création de néologismes dans les nouvelles sciences (Pavel 1993 ; Kokourek \& Rousseau 1992). Si ces approches abordent le même problème de plusieurs optiques différentes, les processus d'apprentissage des structures discursives, et le rapport entre ces structures et les propriétés lexicales du discours scientifique restent encore largement inconnus.

Certaines études ont déjà mis en application des descriptions détaillées fondées sur des corpus importants. Kreztenbacher (1991) a étudié l'ensemble des caractéristiques grammaticales d'un corpus d'abstracts. Salager-Meyer (1994) et Hyland (1994) ont exploité des ensembles importants de textes pour examiner la modalité phrastique (le "hedging ") dans l'anglais académique. De même, Williams (1996) a démontré l'utilité de l'ordinateur dans l'analyse des contextes de certains verbes de l'anglais médical. Mais en général, les études socio-rhétoriques n'ont pas encore exploité la notion de collocation ou de phraséologie dans leurs analyses. De même, les terminologues n'ont pas encore exploité les notions linguistiques de développement textuel et contextuel dans leur méthodologie.

Dans cet article, nous proposons donc de combiner l'approche relativement systématique élaborée par les terminologues avec l'approche contextuelle de l'analyse du discours. 
Ainsi notre but est de démontrer le rôle discursif de la phraséologie dans le discours spécialisé et de rendre compte que la phraséologie occupe une place aussi importante dans l'apprentissage des langues de spécialité que la rhétorique et la terminologie.

\section{La collocation dans les textes spécialisés}

4 C'est dans l'analyse du contexte que l'ordinateur nous permet d'aller bien au-delà des premières analyses de texte. Dans l'élaboration de dictionnaires de langue générale, Sinclair (1991) a démontré que les propriétés globales du langage nous sont pour la plupart cachées et qu'une analyse totale d'un échantillon représentatif de la langue rend compte du rôle primordial du lexique dans la structuration des phrases et dans la différentiation des lexèmes. Le principe «idiomatique » de Sinclair affirme que le mot n'est aucunement sélectionné dans le vide, mais dépend d'une structure syntagmatique plus importante. Le principe s'applique autant aux "collocations" (des mots qui se combinent seulement dans certains contextes, par ex. "auburn hair », "bras ballants ») qu'à des ensembles qui dépassent les limites phrastiques traditionnelles. Par exemple, en français journalistique chaque occurrence de l'expression circonstancielle «pour_s'en_convaincre » est introduite exclusivement par «il_suffit » (le trait _ indique un lexème fixe) :

1. Il_suffit de consulter les parents pour_s'en_convaincre.

2. Il_suffit d'observer l'oisiveté, l'égoïsme et le gaspillage de certains pour_s'en_convaincre...

3. ..., il_suffit_pour_s'en_convaincre, de rappeller que... l'économie française avait détruit (comme disent les experts) des emplois par centaines de milliers.

4. Secundo, on rappellera que les enfants et les animaux sont très largement utilisés par la publicité - la contemplation d'un écran suffit pour_s'en_convaincre.)

5. Il n'était que de voir l'embarras visible du secrétaire au trésor interrogé le 24 janvier à l'occasion d'un magazine télévisé pour_s'en_convaincre.)

5 Ces extraits, tirés d'un corpus du Monde d'environ 1000000 mots (Gledhill, à paraître), et même les quelques exceptions (dont 4 et 5), illustrent l'emploi très régulier d'expressions redondantes ou "pré-fabriquées" qui servent à orienter le discours et à rendre la proposition symétrique du point de vue syntactique. La phrase "pour s'en convaincre » n'est pas strictement obligatoire du point de vue syntactique mais elle fonctionne comme une «locution» dans le sens où elle fait partie d'un ensemble lexical unitaire, bien que séparé structuralement du syntagme verbal. Nous ne considérons pas l'expression comme un ensemble sémantique mais plutôt un ensemble qui joue un rôle rhétorique bien précis.

6 Le terme "phraséologie» est souvent utilisé pour recouvrir non seulement des collocations de base qui sont simplement des mots reliés comme sans pour autant, en somme, not to mention, just in case (des locutions et des expressions figées : Gross 1996) mais aussi les chaînes lexicales qui impliquent des continuations assez longues, telles the fact that, for the very simple reason that, c'est pour ça que, c'est là où... souvent évoquées par les termes « formule » ou « groupement discursif » (Mochet 1997). La phraséologie n'est donc pas comparable aux niveaux lexicaux ou syntaxiques mais appartient au système des expressions d'une communauté : un élément fondamental de la rhétorique. Makkai (1992) a remarqué que ces ensembles sont beaucoup plus courants dans la langue que les 
idiotismes traditionnels. Avant l'arrivée massive des corpus informatisés, Yorio (1980) et Pawley et Syder (1983) avaient préconisé l'analyse des expressions figées chez les apprenants de langues étrangères pour explorer ce qu'ils considéraient être un phénomène fondamental de la compétence linguistique. Plus récemment, Granger (1996) a souligné l'importance de l'identification de ces ensembles et les difficultés que ces expressions présentent aux apprenants. L'exploitation d'un corpus s'impose donc pour l'analyse de ce phénomène dans la langue de spécialité.

\section{Le corpus spécialisé}

7 Un corpus spécialisé consiste en un ensemble de textes qui représentent soit le même registre communicatif, soit le même domaine thématique. En effet, le corpus spécialisé s'avère peut-être plus cohérent que le corpus de la langue générale parce qu'il est lié très facilement à un contexte spécifique. Pourtant, pour bien exploiter le corpus spécialisé, il faut le relier à sa communauté. Avant d'aborder la construction du corpus, nous avons consulté quinze cancérologues à l'université d'Aston en Angleterre. Ceci nous a permis d'établir une base de données qui révèle les intérêts de ce groupe du point de vue local et institutionnel, mais aussi du cadre scientifique global dans lequel il se situe, car ces chercheurs ont des buts différents et appartiennent à des communautés diverses. Le corpus constitue donc un ensemble de textes recueillis suivant trois dimensions textuelles :

8 1. Cohésion interne : il faut représenter les textes produits par le groupe de quinze experts. Nous avons ainsi recueilli dix textes (certains chercheurs publiaient leurs textes en équipe). Ces textes variaient des plus généraux (de Nature et Trends in Pharmaceutical Science aux plus spécialisés: Tetrahedron Letters). Trois textes étaient publiés dans le Journal of Chemistry: Perkin Transactions, pour des raisons historiques liées aux préférences de publication de certains individus.

9 2. Cohésion externe : il faut représenter les textes auxquels les scientifiques sont exposés: que ce soient les textes qu'ils lisent ou les textes qui se réfèrent à leur recherche. Les scientifiques nous ont indiqué les articles qu'ils considéraient comme proches de leurs propres recherches et les articles qui les avaient cités. (Ce procédé nous a fourni 50 textes, dont les périodiques les plus courants sont Carcinogenesis, et Cancer Chemotherapy and Pharmacology).

10 3. Cohérence globale : il faut aussi représenter le non-dit, les textes et les périodiques prestigieux qui traitent du cancer, mais qui ne font pas référence aux intérêts bien particuliers des quinze experts. (Ainsi nous avons sélectionné 90 textes de divers périodiques très prestigieux qui traitent $\mathrm{du}$ cancer ou l'oncogenèse : par exemple: International Journal of (ancer et le British Medical Journal).

11 Le corpus entier se compose donc de vingt-deux périodiques, 150 textes, 500000 mots (nous employons le sigle PSC: Pharmaceutical Sciences Corpus). Cette approche heuristique présente plusieurs avantages. Le PSC ne représente pas une communauté institutionnelle, mais reflète la variété de lecture possible d'une telle communauté. Le corpus touche à la communauté discursive globale (y compris les textes les plus prestigieux sur le cancer) sans se concentrer sur une maladie ou un thème en particulier (des textes parlent de l'étiologie, du traitement régional des patients, des guérisons non conventionnelles, de la chimie structurale et organique...). Nous pouvions consulter les 
auteurs directement, découvrant les buts spécifiques de leurs textes et les liens entre leurs travaux et les tendances globales de la recherche cancérologique. De plus, les chercheurs les plus expérimentés assuraient la rédaction de périodiques très prestigieux, et ils avaient des remarques pertinentes quant au langage et à la phraséologie qu'on trouvait dans leurs propres productions.

Le résultat est que nous accédons à la fois à un corpus cohérent et à un discours particulier, lié à une époque particulière, mais qui représente une variété de spécialités qui font référence au cancer. Par exemple, un jeune chercheur se disait " cancérologue " seulement dans le sens où ses recherches allaient se révéler pertinentes à un ensemble de maladies génétiques. Ses textes sont aussi importants que les textes des bases sur le cancer : encore plus si nous considérons qu'aucun des textes n'est proprement dit «à propos du cancer ». En plus, le cancer est un ensemble de maladies pour lesquelles les chercheurs mêmes avaient des définitions bien différentes par rapport à leurs spécialités. Nous avons donc constitué un corpus qui se définit par rapport à la référence au cancer, et donc à un ensemble d'activités de recherche, et non par rapport à la maladie même.

\section{Exploitation du corpus}

Pour aborder le corpus de façon systématique, nous avons identifié les caractéristiques spécifiques de chaque section de l'article spécialisé. Nous avons séparé chaque section (Titre, Abstract, Introduction, Méthodes, Résultats, Discussion) et employé le logiciel Wordlist (Johns - Scott 1994) pour comparer le lexique d'une section par rapport au corpus entier. Ceci nous indique les mots les plus statistiquement «saillants». Pour démontrer une analyse possible du corpus, nous comparons l'emploi de deux mots grammaticaux (une préposition/complémenteur, « to », un pronom/déterminant « this ») et deux termes lexicaux (patient et effect). "To » est significativement fréquent dans les Introductions et les Discussions, et nous l'analysons en tant que mot grammatical typique qui fait ressortir les tendances phraséologiques, sémantiques et rhétoriques du corpus. Par contre, «this » s'avère très significatif dans les Abstracts et les Discussions, et nous l'examinons pour démontrer la reformulation des idées. Les deux termes lexicaux représentent les deux dimensions sémantiques fondamentales du corpus : l'orientation technique et clinique par rapport à l'orientation empirique et scientifique.

\subsection{La phraséologie d'un mot grammatical typique : « to »}

14 Tout mot grammatical dans le corpus révèle une phraséologie plus restreinte que l'ensemble de ses emplois dans la langue générale. En comparant les emplois classés dans le dictionnaire Collins-Cobuild (Sinclair 1991), nous trouvons qu'en général les mots grammaticaux les plus fréquents retiennent moins d'un tiers de leurs emplois dans le corpus (Gledhill 1995).

Hormis les locutions figées (according to, in addition to) «to » ne garde pas son rôle de préposition classique dans le corpus. Plus généralement, il est impliqué dans des propositions complétives, telles it is important to, have been found to, are designed to qui sont fréquentes dans les Introductions tandis que les complétives en "that» (The possibility that, it has been found that) se trouvent plus dans les Abstracts et les Discussions. En général, on peut accorder une fonction active aux expressions complétives en « to » et une fonction " abstraite » aux expressions en « that », ce qui semble établir une différence 
rhétorique de base entre l'Introduction et le reste du corpus. Dans les Introductions on trouve par exemple des explications en "known_to", ce qui s'exprime plutôt par "It is known that » dans les Discussions.

Cells are known to bind p. 53

chemicals are known to cause embryotoxicity enzymes inhibit hepatic MFO activity

hydrolysis is known to proceed via a 2-step reaction proteins are known to repair the 6-0 methylguanine

A la différence de "known_to", l'expression "appears_to" s'emploie toujours dans des contextes négatifs, souvent avec une proposition subordonnée qui contredit la proposition principale :

Although the regulation of MyoD1 is not fully understood, this appears to perform critical functions.

However, the function of $\mathrm{p} 52 .$. does not appear to stimulate DNA synthesis directly.

Many tumours appear to have no relation to DNT oncogenic viruses However, this appears to contradict some of our preliminary observations.

It appears to be an ubiquitous protein, although there is no correlation...

16 Ces occurrences démontrent que les Introductions ne tendent pas à exprimer la modalité directement et que loin d'être simplement «modale» l'expression "appears to » enclenche une phraséologie plus longue qui implique la comparaison et la contradiction d'observations empiriques.

Comme nous l'avons souligné plus haut, dans toute la phraséologie du corpus, une distinction sémantique s'établit : l'orientation de recherche (centrée sur l'expression de la recherche même ou de son organisation empirique) et l'orientation technique (centrée sur les procédés biochimiques ou cliniques). Par exemple, l'ensemble «able_to» permet l'expression des possibilités de recherche (à noter: les verbes qui décrivent la participation active des chercheurs en italique) :

we were able to compare the patterns

we are able to confirm that...

if we were able to design an interim system

we are not yet able to give a definitive statement

In 16 cases we were able to identify the structural defects

Quand il s'agit de sujets biochimiques, en revanche, les verbes correspondent à des termes techniques ayant un rapport avec des procédés biochimiques :

agents that are able to down regulate

gangliosides are able to function as cells

human IL2 is not able to induce an immune response

the most potent of these is not able to maintain cAK III

The...analogous tumor was also able to metastasize.

19 Ayant admis cette distinction de base, on note qu'elle s'applique parfois de façon plutôt étonnante. Par exemple, dans l'expression «lead_to », la forme au passé (led_to) se réserve exclusivement aux sujets orientés vers la recherche :

These observations led to comparative studies these findings led to widespread use of hormonal aspects.

Identification of cell response led to the investigation of radioimmunization.

we describe the rationale which led to speculation that $5 \mathrm{HT} 3$ receptors. 
These results led to the selection of a battery of immune assays.

Tandis que l'orientation biochimique s'exprime uniquement au présent (leads_to) :

response to DNA damage leads to an arrest of the cells

This [process] in turn leads to increased conversion of the lactase

This process leads to inhibition of intracellular concentrations

altered membrane transport leads to degradation extracelluar matrix (ECM)

the agonist 2-methyl 5HT leads to release of substance $\mathrm{P}$

Cette distinction selon le temps verbal s'accorde partiellement avec une conception du présent comme «temps de l'explication» et le passé comme «temps de l'action scientifique ", mais ne s'accorde pas avec d'autres verbes dans notre corpus, comme nous le verrons plus bas. D'ailleurs nous trouvons une distinction similaire avec les passifs dans les Méthodes, mais non pas avec les passifs ailleurs. Il semble que le temps dépende en partie d'une phraséologie liée à certaines expressions figées.

Enfin, la phraséologie la plus importante de «to» dans les Introductions concerne sa collocation avec «was » pour exprimer les buts de l'article. La variété des expressions possibles mérite qu'on en cite plusieurs :

\author{
The aim of this study was to compare \\ The intention was to determine \\ One further goal was to evaluate \\ The key to the plan was to examine \\ Therefore our second objective was to expand data \\ Our purpose was to explore whether \\ Another goal of these studies was to identify DNA adducers \\ The aim of the present series of these studies was to investigate \\ The goal of this study was to re-evaluate \\ A main task was to study whether ... \\ Thus, the first aim of the present study was to test... \\ The purpose of the Bristol 3rd stage trail was to use a new model... \\ The purpose of this work was to widen the research window... \\ The purpose of the current report was to *generate and trap*...
}

On peut noter que l'ensemble « was_to " n'est pas classable comme élément constituant grammatical classique, et pourtant cette expression constitue le seul élément constant dans ces expressions. Si le seul critère d'identification des lexèmes collocatifs réside dans le figement, nous sommes tenus de considérer "was_to » comme élément fondamental de l'expression. Tandis que le noyau de l'expression est fixe, l'expression des buts de la recherche même peut varier très largement jusqu'à des formules du type "verbe délexicalisé »: widen the research window, use a new model. Une exception se présente pourtant: generate and trap, ce qui ressemble à un procédé clinique ou biochimique. Cette exception s'avère particulièrement intéressante: elle prouve qu'on peut s'exprimer autrement. En fait il n'y a pas de raison que l'on ne puisse employer l'expression « the aim ...was_to» (+ procédé clinique/biochimique). Mais la prépondérance de l'expression de procédés de recherche nous indique que la phraséologie se fixe autour d'un éventail sémantique limité. Il faut aussi considérer la dimension rhétorique : il se trouve que dans les Introductions, on préfère l'expression des buts en tant qu'objectifs de recherche nouveaux plutôt que de nouvelles méthodologies ou des détails de procédés biochimiques. 


\section{2. «This » et la reformulation textuelle}

23 Si l'emploi de "to » dans le corpus nous démontre les spécificités phraséologiques de certaines sections rhétoriques, la phraséologie de «this " dans le corpus nous permet d'explorer un autre domaine textuel : celui de la reformulation. Naturellement, l'emploi du mot implique toujours une référence anaphorique. Nous voyons que cette référence s'opère sur le plan des deux dimensions sémantiques évoquées plus haut. Par exemple, avec des substantifs qui reformulent des résultats, la préposition «this » se combine toujours avec « in » :

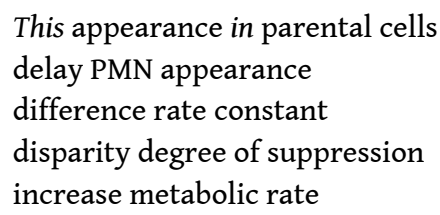

En revanche, avec les substantifs qui reformulent des groupes d'observations plutôt que des résultats globaux, on note l'emploi consistant de la préposition « of » :

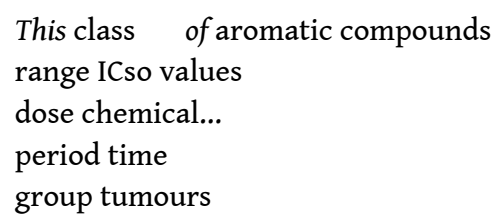

Cette liste omet pourtant le mot lexical le plus employé comme reformulateur : «effect ». Le mot «effect » est unique puisqu'il peut faire référence autant à des entités ou processus biochimiques qu'à des observations empiriques. Comme les mots dans les exemples qui précèdent, « effect » englobe les concepts auxquels il faiit référence tout en les classifiant en tant que phénomènes observables et mesurables (en français: une motion... $\rightarrow$ cet effet, une réaction... $>>$ cet effet). On peut ainsi considérer qu'il s'agit d'un remodelage des résultats entrepris par l'auteur. En analysant le contexte, on trouve que "effect " s'emploie dans une phraséologie plus large permettant une évaluation ou un commentaire sur les résultats de la recherche tout en y rajoutant de nouvelles observations, comme dans les exemples qui suivent :

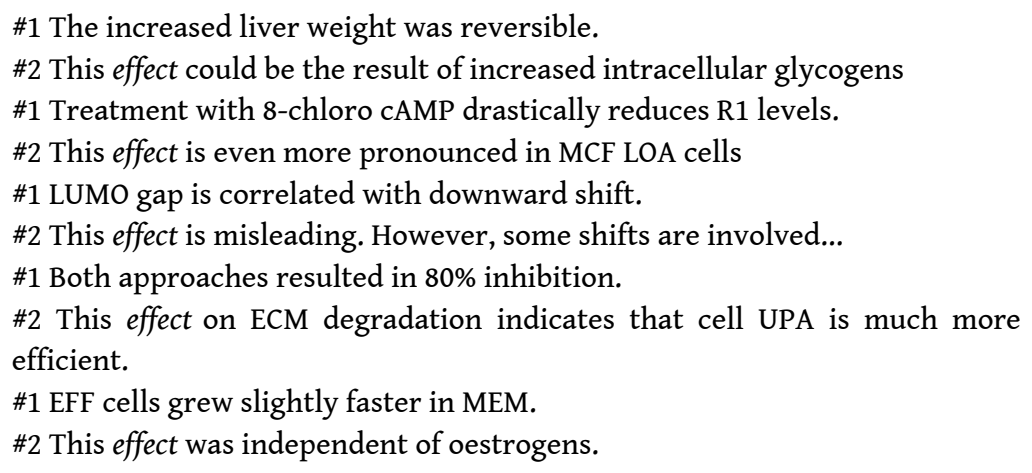

Cependant, «this_result» reformule les résultats de façon explicite en les comparant directement avec l'hypothèse spécifique de chaque article. À la différence de la phraséologie en "effect», les expressions qui suivent "this result» expliquent la signification des résultats sans ajouter des observations empiriques nouvelles : 
\#1 DNA sequencing of the melanoma revealed that p53 codons... were wild type.

\#2 This result eliminates the possibility that mutations are germline... it suggests a mutagenic mechanism.

\section{\#1 We observe several large AJ-IX positive mRNAs}

\#2 This result may indicate that AJ-IX is a very distant exon.

\#1 90\% of the carbonium ion was trapped and

\#2 this result suggests that inorganic phosphate can compete with water to trap the ion.

\#1 The reaction.. produces MeOArc.

\#2 This result is consistent with the partitioning of a common intermediate.

\#1 The study... produced a 23 response rate

\#2 but we have not been able to reproduce this result.

Il faut remarquer que ces phraséologies ne peuvent pas fonctionner sans qu'il y ait à l'arrière-plan un développement textuel particulièrement compliqué. Par exemple, dans un texte sur la chimie structurale, le terme «synthèse totale » est introduit dans le titre comme le thème principal de l'article ; il s'agit d'une réaction destinée à convertir tous les constituants chimiques engagés dans la réaction :

Titre : ...Total synthesis of $\mathrm{d} 4 \mathrm{~T}$.

Cette idée est élaborée dans un syntagme nominal plus long dans l'Abstract dans lequel on rajoute de l'information à propos des fonctions de la composante chimique, mais où le sens de « synthèse » est toujours conservé :

Abstract : The total synthesis of the antiviral agent $\mathrm{d} 4 \mathrm{~T} .$.

Dans l'Introduction, le terme synthesis est décomposé et reformulé de façon verbale, là où les auteurs ont besoin d'expliquer les procédés spécifiques de leur méthodologie :

Introduction: ...no synthesis to date has commenced from the non-chiral pool materials... We now report that $\mathrm{X}$ alcohol can also be elaborated in six steps to the anti-HIV agent $\mathrm{d} 4 \mathrm{~T}$.

Ensuite dans les Méthodes, la synthèse est reformulée en procédé technique précis :

Méthodes :... Deacetylation was effected [=réalisée]] in quantitative yields by treatment with ammonia to provide $\mathrm{d} 4 \mathrm{~T}$.

Dans les Résultats, nous constatons les premiers emplois de "this», dans son rôle classique. Ici le procédé chimique est reformulé en employant un néologisme « route ». À noter aussi que cet emploi fait référence au processus en employant un verbe spécifique introduit dans les Méthodes, «provides».

Résultats : This route thus provides an anomeric mixture of $\mathrm{d} 4 \mathrm{~T}$.

Finalement, c'est dans la Discussion que la reformulation explicite intervient. D'abord le procédé originel est réintroduit, puis il est reformulé en tant que "méthodologie » et ensuite par un terme encore plus affirmé « stratégie » :

Discussion : ... this total synthesis... establishes this methodology as a general and versatile strategy. 
constatons qu'à partir d'un seul énoncé, en l'occurrence "this ", nous sommes capables d'identifier des processus de création, de décomposition et de reconstitution des concepts à l'intérieur des textes scientifiques. La reformulation n'est donc pas seulement la substitution d'un mot par un autre, mais une trame textuelle avec un sens déterminé et une phraséologie bien définie, au moins dans les textes d'exposition scientifique. Ce processus est aussi évident dans les phraséologies associées aux mots lexicaux.

\section{3. "Patients » et « Effect » : la phraséologie des mots lexicaux}

Nous avons suggéré que la distribution particulière des mots grammaticaux relève du fait que la phraséologie est bien marquée pour chaque section de l'article. Cette variation stylistique est d'autant plus remarquable chez les mots lexicaux. Nous avons aussi suggéré que certains mots font ressortir les grandes tendances rhétoriques du texte. Pour tenter de faire une analyse équilibrée entre mots grammaticaux et mots lexicaux, nous avons repris les contextes les plus typiques de deux mots particuliers : patients et effect. Leur distribution est mieux visualisée quand on les compare avec des mots de sémantique similaire (patients, cancer: mots de désignation clinique, biochimique, cause/effect mots de désignation empirique). Voir tableau 1.

Tableau 1

\begin{tabular}{|l|l|l|l|l|l|}
\hline & Cancer & Patients & Cause & Effect & [Corpus] \\
\hline \hline Titres & 13 & 3 & 0 & 9 & 2123 \\
\hline \hline Abstracts & 55 & 63 & 2 & 55 & 29283 \\
\hline Introductions & 140 & 69 & 24 & 80 & 60809 \\
\hline Méthodes & 50 & 108 & 4 & 53 & 113089 \\
\hline Résultats & 63 & 135 & 13 & 202 & 123084 \\
\hline Discussions & 164 & 130 & 32 & 194 & 114205 \\
\hline Corpus Total & 485 & 508 & 65 & 593 & {$[513931]$} \\
\hline
\end{tabular}

Des concordances sélectives sont présentées dans les Annexes pour démontrer les collocations les plus significatives des deux mots pour chaque section de l'article. Dans le texte qui suit, nous résumons les tendances globales et les alternances d'emploi de ces mots lors de chaque section.

La référence aux patients ne figure presque pas dans les titres. Dans les Abstracts par contre, le mot est très présent et on y remarque une phraséologie bien particulière. Le terme patients est modifié par des propositions relatives (en « who ») qui introduisent des expressions actives où les patients "reçoivent » ou "participent » à des expériences. Cette phraséologie révèle une tendance globale du texte scientifique : on ne donne pas des drogues aux patients, on ne les observe pas directement. À la place, c'est aux patients de " souffrir ", " témoigner de la stabilité » ou " démontrer une bonne progression ». Les 
patients "ont» des caractéristiques que les chercheurs ont remarquées, mais l'expression de cette observation n'est jamais employée dans ce contexte. Par contre, dans les Introductions, cette phraséologie devient moins importante et on emploie des expressions figées, mais plus longues : "phénomène $\mathrm{X}$ in patients with maladie $\mathrm{Y}$ ». Dans les Méthodes, une phraséologie différente s'établit. Il s'agit de deux configurations particulières : les patients ne sont plus des sujets de verbes actifs, mais de verbes passifs. Il s'agit de procédés empiriques pour la plupart: ils sont classifiés, exclus de certains groupes, guéris, etc. La seconde phraséologie des Méthodes rend les patients plus actifs encore: ils «reçoivent» ou "répondent » encore à certains traitements. L'emploi du verbe « exhibit » (=témoigner de) y est particulièrement marqué. Pour dix expressions du genre « receive drug $X$ » nous n'y retrouvons qu'un du type passif « were given drug $X$ ».

Dans les Résultats, l'emploi de patients s'oriente autour de trois nouvelles phraséologies, toutes impliquées dans la quantification des résultats: le «nombre» de patients observés, une phraséologie figée autour de «in (une quantité) patients_treated at » (un dosage particulier) et finalement une nouvelle version de "patients qui reçoivent », mais au participe présent (+observation) « in patients receiving (drug X)». Finalement, les patients sont moins actifs dans les Discussions, où le mot sert à classifier des ensembles qui thématisent les innovations ou applications des articles (management, survival, cancer growth) au lieu des drogues ou des maladies. La variation de la phraséologie dans le corpus suggère, comme pour d'autres mots que nous avons examinés, que ces formes sont exploitées pour des raisons rhétoriques variables.

Le mot "effect " présente deux tendances globales dans le texte, bien qu'il s'agisse d'un des mots lexicaux les plus fréquents et les plus complexes du corpus. En général, le terme est impliqué dans la construction de syntagmes nominaux en « of " (l'effet de) et en « on » (l'effet sur). Mais cet emploi diminue dans les sections ultérieures de l'article par rapport au rôle reformulateur de "this effect», que nous avons évoqué plus haut. Exceptionnellement, le mot s'emploie aussi dans le même sens qu' « affect » (il s'agit d'un verbe terminologisé pour exprimer la mise en application d'un procédé chimique: Deacetylation was effected in quantitative yields).

Dans les Abstracts, l'expression normale des « effects » témoigne de la tendance à discuter des effets "anti-cancer» et donc positifs de certaines drogues. Sa forme principale consiste en une cooccurrence avec en " of " (anti-tumour, growth-inhibitory effect of... (drogue $\mathrm{X})$ ), et on voit dans ce type d'expression que l'effet est déjà considéré comme établi. Le mot s'emploie aussi avec « on » pour établir les effets de certaines drogues dans le syntagme verbal : « Drug X has a (well-documented / dose-dependent / enabling...) effect on (disease $\mathrm{Y}$ ) . Cette expression est aussi fréquente dans les Introductions, mais le mot ne témoigne d'aucune prémodification nominale : les effets des drogues ne sont pas a priori connus dans les Introductions et restent non-prouvés. Dans les Méthodes, le mot s'emploie moins souvent et se limite à l'usage sans déterminant qu'on voit dans les titres " Effect(s) of (traitements/guérisons X) in (maladie Y) ». Son emploi se fige également autour de certains mots composés terminologiques très fréquents qui ont des rapports méthodologiques: dose-effect, median-effect. Dans les Résultats, le mot sert de base d'observations avec des collocations assez restreintes indicate/s, shows + a (similar) effect. Enfin, le mot sert dans les Discussions de reformulateur général prémodifié par des termes spécifiques «This (bimodal, differential...) effect » + prédicat. Cette reformulation permet d'utiliser le mot comme support pour introduire le nouveau terme spécifique, et il 
est remarquable que cette fonction ne se fait pas pour d'autres termes que nous pouvons qualifiés d'« anaphoriques » (this result, this type, this model etc.).

En somme, le mot « effet » nous a fourni beaucoup de données simplement parce qu'il s'agit d'un mot unique qui est exploité avec une régularité étonnante dans ces textes. Il sert de mot charnière et de support nécessaire à la reformulation, surtout pour pouvoir transformer de simples données en hypothèses. Ce procédé est connu chez les linguistes systémiques (Halliday \& Martin 1993) où ils identifient en effet la nominalisation et la métaphore grammaticale comme mécanismes primordiaux de la construction des textes scientifiques. La progression des idées, le cumul et la déconstruction de nominaux que nous avons observés plus haut servent à illustrer que ces processus sont entièrement liés à la phraséologie restreinte d'une poignée de mots. Nous suggérons que ces termes sont en effet de véritables institutions qui servent à réorienter le discours.

\section{Conclusion}

Nous avons essayé de démontrer que même des mots grammaticaux de la langue spécialisée peuvent avoir des collocations très précises, et que ces collocations jouent un rôle important dans la phraséologie de chaque section de l'article. Nous avons suggéré aussi que les cooccurrences des mots lexicaux connaissent une progression particulière dans le texte. Ce qu'il faut retenir dans cette analyse, c'est que ces cooccurrences sont relevées de textes souvent très différents du point de vue thématique et générique. À notre avis, ces cooccurrences témoignent de la présence d'un discours, ou même d'un métadiscours, qui s'accorde avec les activités des chercheurs et impose des régularités parfois étonnantes, voire arbitraires.

Nous avons pu identifier des figements liés à des catégories aussi importantes que le temps, les sens sémantiques des verbes, la négation et la modalité, voire la distribution grammaticale des expressions figées telles in vitro (épithète devant le nom) et in vivo (complément circonstanciel) (Gledhill 1996). Comment expliquer la régularité et la prévisibilité de ces formules? Nous imaginons plusieurs possibilités. Les caractéristiques linguistiques de ces textes représentent très souvent des choix lexicaux restreints, délimités dans un éventail phraséologique potentiellement beaucoup plus large dans la langue générale. La langue spéciale, comme l'a suggéré Sager et alii (1980) tend en effet à figer le discours. Mais si ce figement est connu depuis longtemps, personne n'aurait soupçonné qu'il s'agirait d'un figement dans presque tous les domaines grammaticaux.

Il nous semble que la redondance dans la langue générale joue un rôle dynamique : elle permet une variation libre qui est exploitée au moment venu dans les textes à caractère général. Il s'agirait d'une fonction discursive. Nous proposons que le discours spécialisé se serve de cette redondance autrement. Nous suggérons trois fonctions possibles de la phraséologie dans le discours scientifique:

1. une fonction communautaire

2. une fonction textuelle

3. une fonction génétique

1. La communauté, qui est peut-être autant composée d'anglophones de langue seconde que de langue maternelle préfère certaines structures fixes aux dépens d'autres. Nous signalons que la plupart des articles dans notre corpus ont plusieurs auteurs, souvent de provenance non anglophone. La recherche sur l'emploi et l'apprentissage des expressions 
figées (Granger 1996) a démontré que les apprenants préfèrent exploiter des expressions figées plutôt que des expressions " innovatrices ", et ce serait le cas de notre corpus. En plus, la prévisibilité du discours mènerait à une voix discursive qui se répand dans la lecture générale sans pour autant que ce discours soit "appris». Si le figement représente la cohérence de la communauté discursive, c'est qu'il permet la prévision et l'acceptation dans la communauté. C'est la même fonction que l'«accent» ou le «dialecte» dans les communautés linguistiques plus larges: le figement permet d'identifier les nouveaux venus, et comme l'a montré Myers (1990) permet aussi l'exclusion. Nous avançons donc que la phraséologie est un aspect très largement inconscient et acquis du discours scientifique.

2. La phraséologie joue un rôle déterminant dans la définition du "genre » textuel. Nystrand (1986) a souligné le fait que la lecture et la production des textes ne sont pas «linéaires ». Dans notre enquête nous avons trouvé que les scientifiques ne lisaient pas tout le texte, choisissant des points d'entrée correspondant à des questions formulées lors d'une première lecture de l'Abstract. Une fonction simple de la phraséologie prévisible serait donc de réduire la possibilité d'expressions ambiguës et de signaler clairement des références rhétoriques très communes (l'explication des buts, l'élaboration des procédés cliniques répétitifs, l'évaluation des résultats, etc.). En analysant la reformulation dans toutes les sections des textes, ce qui nous a frappé c'est que les textes scientifiques refondent les mêmes idées principales lors du texte : la Discussion ressemble en effet à un résumé reformulatif, les Résultats reprennent les mêmes expressions des Méthodes, etc. Parfois on reformule en élaborant les termes-clés, parfois en les englobant par des termes plus généraux, souvent ayant un rapport avec la proposition revendiquée (the " claim »). Pourtant, chaque section de l'article contient l'essentiel de l'argument : en reformulant, les auteurs mettent leurs idées en scène au début et les déconstruisent dans le reste de l'article. Comme nous l'avons signalé dans les sections précédentes, cette fonction est bien connue dans la littérature linguistique : ce que nous prônons, c'est le lien entre la phraséologie et la fonction textuelle.

50 3. La théorie de l'évolution nous apprend que le vrai critère de réussite du gène, c'est le fait d'être reproduit. Au risque de nous voir «contaminer» par le discours de notre propre corpus, nous proposons ici que le phénomène phraséologique correspond aux mêmes règles que l'évolution des fonctions génétiques. Dawkins, et bien d'autres ont remarqué le rôle des "mèmes" dans l'évolution culturelle: la présence d'unités culturelles, souvent apprises dans leur totalité, qui subsistent sans qu'on puisse les expliquer ou même les éradiquer. Par exemple, Dennet (1996) reprend l'exemple des traditions, des refrains musicaux et des proverbes. À bien des égards, les collocations ressemblent à ces entités transmises dans l'évolution du discours. Si Cavalli-Sforza et Felman (1989) ont élaboré une théorie génétique de l'évolution des idées, il ne leur manque que ce concept de collocation pour soutenir leurs observations.

51 La génétique nous apprend aussi que certains gènes fonctionnent dans un contexte précis, mais que dans d'autres contextes ils n'ont pas de fonction et sont redondants. Ce n'est pas la forme du gène qui compte, mais sa mise en application. Évidemment, les collocations et la phraséologie dans le discours obéissent aux mêmes règles. Il y a deux sortes de "réussite» chez les gènes, et probablement chez les collocations aussi : la transmission "heuristique ", qui transmet le gène non parce qu'il fonctionne mieux que d'autres, mais simplement parce qu'il ne présente pas de problèmes pour les individus qui sont contraints de le transporter. En même temps, la transmission « mutative » accorde la 
réussite aux gènes nouveaux ou mutés s'ils arrivent à apporter un avantage au transmetteur.

Dans le contexte du discours scientifique, nous savons que ce sont les «formules toutes faites » et non pas des données en masse que les chercheurs arrivent à faire passer à leurs collègues. Mais, de même pour les gènes « heuristiques ", ce n'est que dans l'application que ces unités retrouvent leur existence. L'application peut, dans le domaine du discours se réaliser simplement dans la "référence » ou la " citation » ou même dans l'application d'une nouvelle perspective, tel l'exemple de la "synthèse " reformulée en "stratégie » que nous avons élaborée plus haut. Il ne serait pas impossible de considérer ainsi les néologismes et les nouvelles combinaisons de collocations. Et nous savons que les calques terminologiques apportent la phraséologie de la discipline originaire, comme l'a démontré Pavel (1993). Dans son histoire sociale de la notion du virus, Kevles (1996) a démontré aussi que ce sont les évolutions de perspectives, liées à des alternances d'expressions qui ont fait progresser la science de la cancérologie. Le virus, dit-il, ne pouvait en aucune publication s'exprimer en sujet de phrase parce qu'on n'acceptait pas cette valence. Selon Kevles, les premiers virologistes ont dû y supplanter le terme moins menaçant « milk factor». Nous associons donc le flux des collocations et de la phraséologie au conflit discursif qui mesure très souvent la réussite scientifique non pas en termes rationnels ou cliniques, mais en citations et en nombre de textes publiés.

\section{BIBLIOGRAPHIE}

Borko, H. \& C.L. Bernier. 1975. Abstracting Concepts and Methods. New York : Academic Press.

Cavalli-Sforza L. et M. Felman. 1989. Cultural Transmission and Evolution. New Jersey : Princeton University Press.

Cremmins E.T. 1982. The Art of Abstracting. Philadelphia : ISI Press.

Dennet, D.C. 1996. Darwin's Dagerous Idea. Evolution and the Meanings of Life. New York : Touchstone Books.

Gläser, R. 1992. «A multi-level model for a typology of LSP genres ». Fachsprache 15/1-2, 18-26.

Gledhill, C. 1995. « Collocation and genre analysis. The discourse function of collocation in cancer research abstracts and articles ». Zeitschrift für Anglistik und Amerikanistik 1, 1-26.

Gledhill, C. 1996. « Science as a collocation. Phraseology in cancer research articles ». In Botley S., Glass J., McEnery T. and Wilson A. (dir.), Proceedings of Teaching and Language Corpora. Lancaster University UCREL Technical Papers Vol. 9, 108-126.

Granger, S. 1996. « Prefabricated patterns of advanced EFL writing: collocations and lexical phrases . In Cowie, A. (dir.), Phraseology. Oxford : Oxford University Press.

Gross, G. 1996. Les expressions figées en français. Paris : Ophrys.

Halliday, M. et J. Martin. 1993. Writing Science: Literacy and Discursive Power. Londres : Falmer Press. 
Harvey, A. 1995. « El fonómeno de la reformulación des discurso cientifico ». Lenguas Modernas 22, 105-112.

Hyland, K. 1994. « Hedging in academic writing and EAP textbooks ». ESP Journal 13/3, 239-256.

Johns, T. et M. Scott. 1994. Microconcord Concordancing Programme. Oxford : Oxford University Press.

Kevles, D. 1997. « Pursuing the unpopular: A history of courage, viruses and cancer in Silvers ». In Robert (dir.), Hidden Histories of Science. Londres : Granta, 69-114.

Knowles, F. 1996. « Lexical cartography in LSP texts ». In Somers (dir.), Terminology, LSP and Translation, Studies in Honour of Juan C. Sager. Amsterdam : John Benjamins.

Kokourek, R., L-J. Rousseau et alii. 1992. «Terminologie, discours et textes spécialisés ». In Actes $d u X^{e}$ Congrès international des linguistes. Québec : Presses Universitaires de l'Université Laval, 187-212.

Kretzenbacher, H.L. 1990. Rekapitulation: Textstrategien der Zusammenfassung von wissenschaftlichen Fachtexten. Tübingen : Gunter Narr Verlag.

MacKenzie, J. L. 1992. « What is Functional Grammar? ». In Actes du XV Congrès international des linguistes. Québec : Presses Universitaires de l'Université Laval, 95-106.

Makkai, A. 1992. « Idiomaticity as the essence of language ». In Actes du XV Congrès international des linguistes. Québec : Presses Universitaires de l'Université Laval, 361-365.

Mochet, M-A. 1997. « Expressions et groupements discursifs de l'oral : questions d'inventaire et propositions didactiques ». Colloque triangle, ENS Fontenay/St Cloud, 7-8 mars 1997.

Myers, G. 1990. Writing Biology: Texts in the Social Construction of Scientific Knowledge. Milwaukee, WI : University of Wisconsin Press.

Nwogu, K. N. 1989. « Discourse variation in medical texts: Schema, theme and cohesion in professional and journalistic accounts ». Thèse non publiée, Language Studies Unit, Aston University.

Nystrand, M. 1982. What Writers Know. The Language, Process and Structure of Written Discourse. New York : Academic Press.

Pavel, S. 1993. « Neology and phraseology as terminology-in-the-making ». In Sonneveld, H.B. \& K.L.Loening dir.), Terminology. Applications in interdisciplinary communication. Amsterdam : John Benjamins, 21-34.

Pawley, A. et F. H. Syder. 1983. « Two puzzles for linguistic theory : nativelike selection and nativelike fluency ». In Richards, J. C. et R.W. Schmidt (dir.), Language and Communication. Londres : Longman, 191-227.

Sager, J. C., D. Dungworth et P. F. McDonald. 1980. English Special Languages: Principles and Practice in Science and Technology. Wiesbaden : Oscar Nadstetter Verlag.

Salager-Meyer, F. 1994. « Hedges and textual communicative function in medical English written discourse ». ESP Journal 13/2, 149-170.

Sinclair, J. McH. 1991. Corpus, Concordance, Collocation. Oxford : Oxford University Press.

Stubbs, M. 1996. Text and Corpus Analysis. Londres : Routledge.

Swales, J. 1990. Genre Analysis: English in Academic and Research Settings. Cambridge : Cambridge University Press. 
Thomson, G. and Y. Yiyun. 1991. «Evaluation in the reporting verbs used in academic papers ». Applied Linguistics 12/4, 365-382.

Williams, I. A. 1996. «A contextual study of lexical verbs in two types of medical research article ». ESP Journal 15/3, 175-198.

Yorio, C. A. 1980. «Conventionalized language forms and the development of communicative competence ». TESOL Quarterly 14/4, 433-442.

\section{ANNEXES}

\section{Annexe 1. Concordance de patients dans les articles de recherche}

\section{Abstracts}

1 among patients and controls. Among patients who had progressive disease, 21/

2 maximum tolerated dose (MTD) for patients who had a good performance status, 3 he 48-h samples were obtained from patients who had received leucovorin plus 4 effect of ondansetron principally in patients who had low excretion of cortisol. 5 trations were highest in the three patients who succumbed to pulmonary toxicit Introductions

1 tissues frequently occur in cancer patients, and depletion of host adipose tiss 2 he mediators of cachexia in cancer patients. As a model of cancer cachexia, we 3 treatment of head and neck cancer patients. In order to obtain further insight 4 apeutic treatment of breast cancer patients in the future, it is essential to e 5 issue and muscle protein in cancer patients is an important parameter determi

\section{Méthodes}

1 for entry into the present study, patients were required to have a histologica 2 asis of the TNM classification, the patients were classified into one of three $3 \mathrm{r}$ to and after ifosfamide therapy, patients were hydrated i. v. with I I norma 4 toxic effects of all prior therapy. Patients were excluded from the study if th 5 any toxicity was documented. Three patients were treated at each dose level an $6 \mathrm{FU}$ at $500 \mathrm{mg} / \mathrm{m} 2$ prior to surgery; 6 patients received a 2-h infusion with leuco 7 sion was started. Additionally, all patients received ondansetron ( $8 \mathrm{mg}$, p.o. $\mathrm{x}$ 8 ee of the Free University Hospital. Patients received an i. v. bolus injection $9 \mathrm{~h}$ days. In the statistical analyses patients responding with 'no' or 'mild' naus 10 atients were enrolled at that dose. Patients who exhibited stable disease or a Résultats

$1 \mathrm{~h}$ age and menopausal status of the patients, tumour size, the number of positiv 
2 rall response rate in all eligible patients was $26 \%$ (9/35; $95 \%$ confidence inte 3 on of survival for the 15 evaluable patients was 92 days (range, 30-568 days). A 4 an approximately equal number of patients was present in each group. Similarl 5 he mean 1 -h concentration in these patients was significantly higher than that 6 comparable with those measured in patients treated at $720 \mathrm{mg} / \mathrm{m} 2$. The $\mathrm{tl} / 2 \mathrm{val}$ 7 ea and vomiting occurred in $50 \%$ of patients treated at $6,500 \mathrm{mg} / \mathrm{m} 2$, and one of $8 \mathrm{~d}$. It is noteworthy that four other patients treated at $6,500 \mathrm{mg} / \mathrm{m} 2$ and another 9 ated at $6,500 \mathrm{mg} / \mathrm{m} 2$, and one of six patients treated at this dose experienced $\mathrm{g}$ 10 mbocytopenia occurred in two of six patients treated at $6,500 \mathrm{mg} / \mathrm{m} 2$; in one pa 11 /time profiles were obtained in 15 patients receiving a dose of $80 \mathrm{mg} / \mathrm{m} 2$, mainl 12, chi2 (1)<0.54, P's>0.26). Among patients receiving ondansetron and placebo, 13 ol excretion Separate analyses for patients receiving ondansetron and placebo 14 points, several samples taken from patients receiving leucovorin and SFU were

15. The mean cortisol excretion in patients receiving dexamethasone was $28.8 \mathrm{pm}$

\section{Discussions}

1 ide to parent drug. Interestingly, patients with high concentration ratios expe 2 need to be devised. Besides, many patients with other types of cancer are not 3 gnificant impact on the survival of patients with metastatic melanoma. Thus, ne 4 drug may play in the management of patients with epidermoid head and neck $5 \mathrm{ma} 180$ or from the ascites fluid of patients with hepatoma, appears to differ $\mathrm{f}$

\section{Annexe 2. Concordances d'effect dans les articles de recherche}

\section{Titres}

1 epatocytes of female B6C3F1 mice. 124 Effect of varying exposure regimens on $\mathrm{m}$ 2 duced Lymphosarcoma Cells' Antitumor Effect of Interferon plus Cyclosporine A f $3 \mathrm{~d}$ into the hepatic artery. Effect of toremifene on antipyrine eliminat 4 Soliton Model Compounds combination effect of vaccination with il2 and il4 cdn 5 are absent in chronic pancreatitis. Effect of exogenous heparin on anchorage $\mathrm{i}$

\section{Abstracts}

1 secondary recipients. The antitumor effect of cells generated by IFN plus CsA t

2 le maintaining the cardioprotective effect of the liposome carrier as suggeste

3 nhibited the tumor growth-promoting effect of TP as shown by $81 \%$ and $80 \%$ tum 4 lot analysis. The growth-inhibitory effect of doxorubicin, daunorubicin, N,N5 amil enhanced the growth inhibitory effect of vindesine on both H69/VDS (x 12.0

\section{Introductions}


1 were mediated primarily through an effect on the male (NTP, 1989; Fail et

$2 \mathrm{~s}$ between two bilayer components, an effect on the hydrolysis kinetics of phosp

3 metic agents have a well documented effect on mild to moderate chemotherapy

4 her granisetron has a dose-dependent effect on gastrointestinal transit and to

51 uences have had a profound enabling effect on research in biology. Until a few

$6 \mathrm{y}$ few studies have investigated the effect of liposome encapsulation on the $p$

$7 \mathrm{r}$ words if hyperthermia changes the effect of Doxo on the microcirculation and

8 onditions [10-12 ]. Therefore, the effect of a low dose of the drug might be pote

9 rat liver model to investigate the effect of toremifene on the elimination of

10 tissue might also be a part of the effect of NDP on the cardiac muscle. Anthr

\section{Méthodes}

1 provides graphic representations of dose-effect curves and the median- effect $p$

2 mity of the experimental data to the median-effect principle of the mass-action

3 fold serially diluted to generate a dose-effect relationship in the cytotoxicit

4 rvival distribution. To estimate the effect of different factors on survival, a

5 stable for at least I month.Fig. 2. Effect of curcumin on PKC activity. PKC pa

Résultats

1 arious cell types. Fig. 3 shows the effect of analogues JBI (D domain), JB2 (C

2 again, biotin reduced the antitumor effect, while cephalexin and sulfathiazole

3 16:0-PC showed the least stimulating effect, with a maximum of $109 \%$ after treat

4 roups. If the ANOVA indicated a time effect within one group, data obtained at $\mathrm{i}$

5 hibition (data not shown). A similar effect of the RIa anti-sense was observed $i$

\section{Discussions}

1 to be dose-dependent. This bimodal effect on tumor-cell growth is compatible w

2 (Fig. 3). How can this differential effect on tumorigenicity vs. experimental

3 itogenic activity of the serum. This effect was independent of oestrogens, insu

4 presence of cholesterol, then this effect was not reflected in change kinetics

5 degradation by HT29 TX66 cells. This effect on ECM degradation indicates that c

\section{RÉSUMÉS}

Nous entendons démontrer que les collocations jouent un rôle important dans la construction des idées scientifiques. Alors que la lexicographie a montré l'importance des phraséologies dans la langue générale (Sinclair 1991), les phraséologies en langue spécialisée n’ont pas reçu le même niveau d'attention. Nous examinons les phraséologies associées à deux tournures grammaticales et à deux unités lexicales dans un corpus de 150 textes de cancérologie et nous suivons les 
variations dans leurs collocations en fonction des catégories constitutives d'un genre, l'article de recherche. Il apparaît que les phraséologies ont un rôle crucial à jouer dans l'argumentation et l'établissement du fait scientifique. Les régularités affirmées de ces collocations, dans une large mesure, échappent à l'attention de ceux qui les utilisent, ce qui laisse supposer que la spécialisation en langue est, dans une large mesure, un phénomène subconscient. Par ailleurs, les phraséologies forment le liant de la communauté scientifique et peuvent être conçues comme l'arrière-plan faisant ressortir les nouvelles expressions. Nous soulignons le rôle des phraséologies au sein de la communauté de discours, leur rôle dans le développement des idées et leur possible fonction « génétique » dans l'évolution du discours scientifique.

We claim here that collocation constitutes an essential process in the construction of scientific ideas. While corpus analysis in lexicography has established the importance of idiom in the language as a whole (Sinclair 1991), less attention has been paid to the analysis of phraseology in specialist texts outside the world of terminology. We examine here the phraseology of two grammatical items and two lexical items in 150 cancer texts and set out their changing collocational patterns throughout the research article genre. We find that phraseology has a pervasive role to play in the argumentation and scientific validity of the text. The pervasive regularity of these expressions remain largely unknown to the writers who use them, suggesting that domain specialisation in language is a largely subconscious phenomenon. At the same time, phraseology gives coherence to the community's means of expression and allows for a backdrop against which new expressions may be contrasted. We emphasise the role of phraseology in the discourse community, its role in the textual development of ideas and its possible 'genetic' function in the evolution of scientific discourse.

\section{INDEX}

Keywords : collocation, corpus linguistics, phraseology, reformulation, scientific discourse Mots-clés : collocation, discours scientifique, linguistique de corpus, phraséologie, reformulation

\section{AUTEUR}

\section{CHRISTOPHER GLEDHILL}

Christopher Gledhill est senior lecturer en français et linguistique à l'université de Saint Andrews en Écosse. Il a enseigné l'anglais de spécialité à l'université d'Aston et y a passé sa thèse de doctorat en 1995 ( «Scientific innovation and the phraseology of rhetoric »). Son premier livre, Phraseology in Science Writing, est à paraître ; il prépare actuellement un second livre sur la phraséologie dans les langues artificielles ainsi qu'un projet interdisciplinaire exploitant des corpus multilingues et diachroniques. Le corpus PSC est à la disposition des linguistes. cgl@eila.univ-paris-diderot.fr 